\section{Ganho de peso gestacional e retenção de peso no pós-parto: dados da coorte de nascimentos de 2015, Pelotas, Rio Grande do Sul, Brasil}

\author{
Gestational weight gain and postpartum weight \\ retention: data from the 2015 birth cohort in \\ Pelotas, Rio Grande do Sul State, Brazil
}

\author{
Ganancia de peso gestacional y retención de \\ peso en el posparto: datos de la cohorte \\ de nacimientos de 2015, Pelotas, \\ Río Grande do Sul, Brasil
}

Thaynã Ramos Flores 1 Bruno Pereira Nunes 1

Vanessa Iribarrem Avena Miranda 1 Mariangela Freitas da Silveira 1 Marlos Rodrigues Domingues 1 Andréa Dâmaso Bertoldi 1

doi: 10.1590/0102-311X00203619

Correspondência

T. R. Flores

Universidade Federal de Pelotas.

Rua Marechal Deodoro 1160, Pelotas, RS 96020-220, Brasil. floresrthayna@gmail.com

1 Universidade Federal de Pelotas, Pelotas, Brasil. longitudinal com dados da coorte de nascimentos de Pelotas, Rio Grande do Sul, Brasil, 2015. O ganho de peso gestacional foi classificado segundo o Instituto de Medicina dos Estados Unidos. A retenção de peso aos 3 e 12 meses foi calculada considerando-se o peso da mãe em cada período, subtraído do peso pré-gestacional. Para identificar as possíveis desigualdades, utilizou-se o Slope Index (SII) e o Concentration Index (CIX). A amostra analitica incluiu as mães com informações para o desfecho em estudo $(n=4.102)$. A prevalência de ganho de peso gestacional adequado foi de 33,5\% (IC95\%: 32, 1; 35,0). Foram encontradas desigualdades discretas na prevalência de ganho de peso adequado entre as menos escolarizadas [CIX = 1,88 (IC95\%: -0,76; 4,52); SII = 4,27 (IC95\%: -0,87; 9,41)] e entre as mães pertencentes ao quinto mais pobre - 1o quinto - [CIX = 1,04 (IC95\%: - 1,60; 3,67); SII = 2,93 (IC95\%: -2,06; $7,92)]$, porém, estas diferenças não foram estatisticamente significativas. A média de retenção de peso pós-parto foi de 2,3kg $(D P=6,4)$ e de $1,4 \mathrm{~kg}(D P$ $=8,8)$ após 3 e 12 meses do parto, respectivamente. Um terço das mulheres apresentou ganho de peso considerado adequado. As desigualdades observadas na prevalência de ganho de peso gestacional adequado com relação à menor escolaridade materna e quinto mais pobre de renda não foram estatisticamente significativas.

Ganho de Peso na Gestação; Gravidez; Índice de Massa Corporal; Fatores Socioeconômicos 


\section{Introdução}

O ganho de peso gestacional é considerado um evento biológico complexo e indispensável para o crescimento saudável do bebê 1 . No entanto, o ganho de peso excessivo durante a gestação pode afetar tanto a mãe quanto a criança 2,3. Existem fatores que podem contribuir para o maior ganho de peso gestacional, entre eles, a idade e a escolaridade materna, intervalo entre as gestações e o estado nutricional pré-gestacional 4,5.

O ideal é que as mulheres tenham um adequado ganho de peso durante a gestação, como por exemplo, para uma gestante com índice de massa corporal (IMC) $\geq 18,5 \mathrm{a} \leq 24,9 \mathrm{~kg} / \mathrm{m}^{2}$, ou seja, eutrófica, o ideal é que o ganho de peso total seja de $11,5 \mathrm{~kg}$ a $16 \mathrm{~kg}$, no máximo 1 . Não obstante, nem sempre essa é a realidade de muitas gestantes, o que pode levar a intercorrências gestacionais e influenciar nas condições de saúde materno-infantil. As principais consequências evidenciadas, na literatura, entre as gestantes com ganho de peso excessivo foram a ocorrência de diabetes gestacional, pré-eclâmpsia, eclâmpsia e complicações no parto 5 . Para as crianças, observou-se maior risco de peso elevado ao nascer e de sobrepeso e obesidade na infância 4,6,7.

Outro evento relacionado com o ganho de peso gestacional é a retenção de peso no pós-parto. De acordo com estudos prospectivos, a prevalência de mulheres que retornaram ao peso pré-gestacional em até um ano após o parto é menor que $65 \%$ 8,9. Muitas mulheres iniciam a gestação com excesso de peso, ou ainda, possuem ganho de peso excessivo ao final do período gestacional 10. Um estudo realizado em capitais brasileiras com aproximadamente seis mil gestantes encontrou prevalência de 19,2\% e 5,5\% de sobrepeso e obesidade, respectivamente 11. A retenção de peso no pós-parto caracteriza-se por ser a diferença entre o peso após o parto e o pré-gestacional 12.

Alguns trabalhos já avaliaram o ganho de peso gestacional e a retenção de peso associados a aspectos demográficos e socioeconômicos 13,14. No entanto, existe uma lacuna importante a ser preenchida, para melhor compreensão da temática. Os estudos destacam que os fatores socioeconômicos, bem como demográficos, podem estar relacionados ao ganho de peso gestacional 15,16, o que ressalta a importância de verificar as possíveis desigualdades no ganho de peso gestacional adequado. Assim, o objetivo do presente estudo foi: (a) descrever o ganho de peso gestacional, de acordo com o IMC prégestacional, e a retenção de peso após 3 e 12 meses do parto; (b) identificar possíveis desigualdades socioeconômicas no ganho de peso gestacional adequado.

\section{Metodologia}

Estudo de coorte prospectiva. Os dados deste trabalho foram coletados nos estudos da Coorte de Nascimentos de Pelotas de 2015, realizados na cidade de Pelotas, Rio de Grande do Sul, Brasil. Todas as mulheres que moravam na área urbana do município e tiveram seus filhos em uma das cinco maternidades de Pelotas, no período de 1o de janeiro de 2015 a 31 de dezembro de 2015, foram convidadas a participar do estudo. Detalhes do estudo podem ser encontrados no artigo metodológico Cohort Profile: The 2015 Pelotas (Brazil) Birth Cohort Study 17.

Para este artigo foram utilizados os dados coletados ao nascimento (estudo perinatal, nas maternidades), aos 3 e 12 meses de vida das crianças. Nos estudos realizados no $3 \underline{o}$ e 12 o meses de vida das crianças, as mães foram entrevistadas nos domicílios respondendo a um questionário padronizado sobre o período após o nascimento 17.

Os desfechos em estudo foram ganho de peso gestacional adequado e retenção de peso aos 3 e aos 12 meses. Para o desfecho ganho de peso gestacional adequado foi usada a variável ganho de peso gestacional, que foi construída por meio da subtração do peso ao final da gestação e peso pré-gestacional, ambos autorreferidos pela mãe no momento da entrevista e, posteriormente, categorizada em: ganho de peso adequado, insuficiente e excessivo, de acordo com a classificação do Instituto de Medicina dos Estados Unidos (IoM) 1. O ganho de peso foi classificado de acordo com o IMC, da seguinte maneira: para aquelas mulheres com baixo peso $\left(<18,5 \mathrm{~kg} / \mathrm{m}^{2}\right)$, o ganho de peso gestacional poderia ser de $12,5 \mathrm{~kg}$ a $18 \mathrm{~kg}$; para as eutróficas $\left(18,5 \mathrm{~kg}\right.$ a $\left.24,9 \mathrm{~kg} / \mathrm{m}^{2}\right)$, de $11,5 \mathrm{~kg}$ a $16 \mathrm{~kg}$; para as com sobrepeso $(25$ a $\left.29,9 \mathrm{~kg} / \mathrm{m}^{2}\right)$; e obesidade $\left(\geq 30 \mathrm{~kg} / \mathrm{m}^{2}\right)$ o ganho poderia ser de $7 \mathrm{~kg}$ a $11,5 \mathrm{~kg}$ e de $5 \mathrm{~kg}$ a $9 \mathrm{~kg}$, respectivamente. A retenção de peso 3 e 12 meses após o parto foi calculada subtraindo-se o peso da mãe em 
cada um destes momentos, do peso pré-gestacional. O peso da mãe três meses após o parto foi aferido por meio de balança portátil digital da marca TANITA, modelo UM-080 (TANITA Brazil, São Paulo, Brasil), com precisão de $100 \mathrm{~g}$ e com capacidade para até $150 \mathrm{~kg}$. Na pesagem realizada 12 meses após o parto foi utilizada balança portátil da marca SECA, modelo 803 (SECA Brasil, Cotia, Brasil), com os mesmos parâmetros de precisão e capacidade da marca anterior. As medidas antropométricas referidas foram realizadas na ocasião das entrevistas.

As variáveis independentes maternas usadas para a descrição da amostra foram: idade (coletada em anos completos e categorizada em: 13-19, 20-24, 25-29, 30-34 e 35 ou mais), cor da pele materna por meio de observação da entrevistadora (classificada em branca, preta e parda), situação conjugal materna (com e sem companheiro ou companheira), escolaridade materna (coletada em anos completos de estudos , categorizada em: 0-4, 5-8, 9-11 e 12 ou mais anos), renda familiar em salários mínimos (analisada em quintos de renda, 1o - mais pobre e 5o - mais rico) e o IMC pré-gestacional, operacionalizado pela divisão do peso pré-gestacional em quilogramas $(\mathrm{kg})$ pela altura em metros ao quadrado $\left(\mathrm{m}^{2}\right)$, categorizado de acordo com o Organização Mundial da Saúde (OMS) $18 \mathrm{em}$ : baixo peso $\left(<18,5 \mathrm{~kg} / \mathrm{m}^{2}\right)$, eutrofia $\left(18,5 \mathrm{a} 24,9 \mathrm{~kg} / \mathrm{m}^{2}\right)$, sobrepeso $\left(\geq 25 \mathrm{a} 29,9 \mathrm{~kg} / \mathrm{m}^{2}\right)$ e obesidade $\left(\geq 30 \mathrm{~kg} / \mathrm{m}^{2}\right)$. As variáveis de escolaridade materna e renda familiar foram utilizadas para as análises de desigualdades, conforme será descrito.

A análise dos dados foi realizada usando-se o programa estatístico Stata, versão 15.0 (https:// www.stata.com). Inicialmente, foi realizada a descrição da amostra apresentando as proporções das variáveis independentes e a média de retenção de peso materno aos 3 e 12 meses pós-parto. Ainda, para verificar as possíveis diferenças estatísticas nas distribuições das categorias de ganho de peso gestacional de acordo com o IMC pré-gestacional utilizou-se o teste de qui-quadrado para heterogeneidade, obtendo-se as prevalências e intervalos de 95\% de confiança (IC95\%). Para identificar as possíveis desigualdades, foram calculados dois índices: o Slope Index (SII) e o Concentration Index (CIX) 19,20. O SII apresenta a diferença absoluta em pontos porcentuais (variando de $-100 \mathrm{a}+100 \%$ ) entre os extremos como, por exemplo, o quinto mais rico e o mais pobre, usando um modelo de regressão logística. O CIX é baseado em uma escala que varia de -1 a +1 , em que zero representa uma distribuição sem desigualdades. Valores CIX positivos indicam que a distribuição é a favor dos ricos. O SII apresenta a desigualdade absoluta e o CIX indica a desigualdade relativa. Considerou-se o nível de $5 \%$ de significância .

O trabalho foi aprovado pelo Comitê de Ética da Escola Superior de Educação Física da Universidade Federal de Pelotas sob o Protocolo 522.064 registrado na Plataforma Brasil. Todas as entrevistas foram realizadas após assinatura, pelas mães, do termo de consentimento livre e esclarecido.

\section{Resultados}

A coorte de 2015 foi composta por 4.275 participantes, no estudo perinatal. A taxa de resposta do estudo perinatal foi de $99,8 \%$, seguida por $96,1 \%$ aos três meses e $95,4 \%$ aos 12 meses de idade das crianças. A amostra analítica deste trabalho incluiu somente as mães dos participantes, sendo 4.098 mulheres elegíveis (excluindo as mães de natimortos) que possuíam informações disponíveis para ganho de peso gestacional. Na Tabela 1, estão as características da amostra. Com relação ao IMC pré-gestacional materno, $28,2 \%$ e $18,8 \%$ das mulheres estavam com sobrepeso e obesidade, respectivamente.

A média de ganho de peso gestacional da amostra foi de $12 \mathrm{~kg}$ (desvio padrão $-\mathrm{DP}=6,7$ ). A prevalência de ganho de peso gestacional insuficiente, adequado e excessivo foi de 30,6\% (IC95\%: 29,2; 32,0), 33,5\% (IC95\%: 32,1; 35,0) e 35,9\% (IC95\%: 34,4; 37,4), respectivamente. A proporção de mulheres com ganho de peso gestacional adequado foi menor quanto maior o IMC pré-gestacional (Tabela 2). As diferenças observadas quanto ao ganho de peso gestacional de acordo com o IMC pré-gestacional foram estatisticamente significativas $(\mathrm{p}<0,001)$. Com relação à retenção de peso três meses após o parto, a média foi de 2,3kg ( $\mathrm{DP}=6,3)$ e, após 12 meses, de $1,4 \mathrm{~kg}(\mathrm{DP}=8,7)$. 
Tabela 1

Características da amostra geral e da amostra analisada aos 12 meses de acordo com o desfecho de ganho de peso gestacional adequado. Coorte de nascimentos 2015, Pelotas, Rio Grande do Sul, Brasil.

\begin{tabular}{|c|c|c|c|}
\hline Variáveis & $\begin{array}{l}\text { Amostra } \\
\text { geral } \\
\text { n (\%) }\end{array}$ & $\begin{array}{l}\text { Ganho de peso gestacional } \\
\text { adequado * } \\
{\left[\begin{array}{c}{[\mathrm{N}=1.374]} \\
\mathrm{n}(\%)\end{array}\right.}\end{array}$ & Valor de $p$ \\
\hline Idade materna (anos) [N = 4.274] & & & 0,28 \\
\hline $13-19$ & $623(14,6)$ & $159(11,6)$ & \\
\hline $20-24$ & $1.008(23,6)$ & $312(22,7)$ & \\
\hline $25-29$ & $1.006(23,5)$ & $350(25,5)$ & \\
\hline $30-34$ & $1.004(23,5)$ & $330(24,0)$ & \\
\hline $35+$ & $633(14,8)$ & $223(16,2)$ & \\
\hline Cor da pele materna $[\mathrm{N}=4.271]$ & & & 0,01 \\
\hline Branca & $3.071(71,9)$ & $1.044(76,0)$ & \\
\hline Parda & $561(13,1)$ & $174(12,7)$ & \\
\hline Preta & $639(15,0)$ & $155(11,3)$ & \\
\hline Situação conjugal materna [N = 4.274] & & & 0,14 \\
\hline Com companheiro & $3.667(85,8)$ & $1.203(87,6)$ & \\
\hline Sem companheiro & $607(14,2)$ & $171(12,4)$ & \\
\hline Escolaridade materna (anos) $[\mathrm{N}=4.274]$ & & & 0,07 \\
\hline $0-4$ & $391(9,2)$ & $107(7,8)$ & \\
\hline $5-8$ & $1.095(25,6)$ & $327(23,8)$ & \\
\hline $9-11$ & $1.458(34,1)$ & $486(35,4)$ & \\
\hline 12 ou mais & $1.330(31,1)$ & $454(33,0)$ & \\
\hline Renda familiar (em quintos) $* *[N=4.273]$ & & & 0,03 \\
\hline 1으 (mais pobre) & $846(19,8)$ & $256(18,7)$ & \\
\hline 20 & $859(20,1)$ & $241(17,6)$ & \\
\hline 3으 & $853(20,0)$ & $316(23,0)$ & \\
\hline 4 은 & $856(20,0)$ & $271(19,7)$ & \\
\hline 5o (mais rico) & $859(20,1)$ & $289(21,0)$ & \\
\hline
\end{tabular}

* Amostra analítica 12 meses pós-parto (acompanhamento dos 12 meses);

** Média de valores (em Reais) de cada quinto de renda familiar: 1 o quinto $=\mathrm{R} \$ 725,17 ; 2$ q quinto $=\mathrm{R} \$ 1.369,10$; 3o quinto = $\mathrm{R} \$ 2.038,80 ; 4$ o quinto $=\mathrm{R} \$ 3.011,86 ;$ ㅇ quinto $=\mathrm{R} \$ 8.133,88$.

Valor de p: teste de qui-quadrado para heterogeneidade entre as amostras.

Tabela 2

Ganho de peso gestacional classificado de acordo com o Instituto de Medicina dos Estados Unidos 1, segundo índice de massa corporal (IMC) pré-gestacional $\left(\mathrm{kg} / \mathrm{m}^{2}\right)$. Coorte de nascimentos, 2015, Pelotas, Rio Grande do Sul, Brasil ( $\left.N=4.098\right)$.

\begin{tabular}{|c|c|c|c|c|}
\hline \multirow[t]{3}{*}{ Ganho de peso gestacional (kg) } & \multicolumn{4}{|c|}{ IMC materno pré-gestacional [\% (IC95\%)] } \\
\hline & $<18,5$ & $18,5 \mathrm{a}<25,0$ & $\geq 25,0$ a $<30,0$ & $\geq 30,0$ \\
\hline & {$[n=154]$} & {$[n=2.039]$} & {$[n=1.169]$} & [n = 779] \\
\hline Insuficiente & $40,5(33,0 ; 48,6)$ & $37,9(35,8 ; 40,0)$ & $17,4(15,2 ; 19,6)$ & $29,4(26,2 ; 32,7)$ \\
\hline Adequado & $40,5(33,0 ; 48,6)$ & $36,1(34,1 ; 38,3)$ & $32,8(30,2 ; 35,6)$ & $26,2(23,2 ; 29,5)$ \\
\hline Excessivo & $19,0(13,4 ; 26,0)$ & $26,0(24,1 ; 27,9)$ & $49,8(46,9 ; 52,7)$ & $44,4(40,9 ; 47,9)$ \\
\hline
\end{tabular}

IC95\%: intervalo de 95\% de confiança.

Nota: valor de $p<0,001$ teste de quiquadrado para heterogeneidade. 


\section{Figura 1}

Prevalência (\%) de ganho de peso adequado e inadequado classificado de acordo com o Instituto de Medicina dos Estados Unidos 1, de acordo com a escolaridade materna e renda familiar em quintos. Coorte de nascimentos, 2015, Pelotas, Rio Grande do Sul, Brasil ( $N=4.098)$.

1a) Escolaridade materna

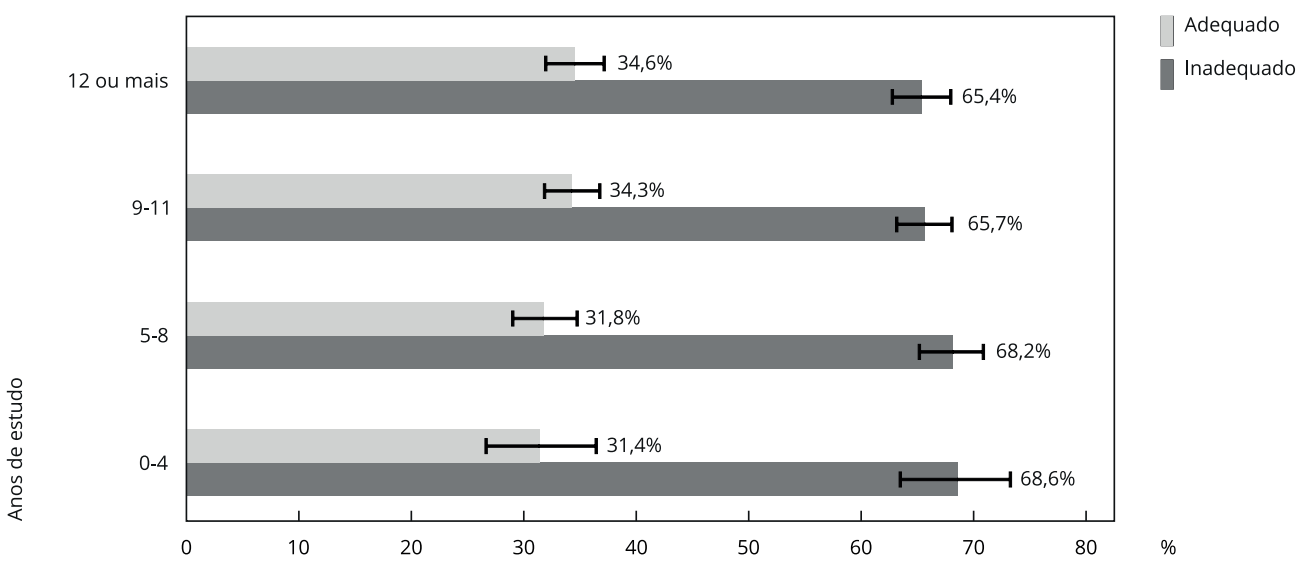

1b) Renda familiar

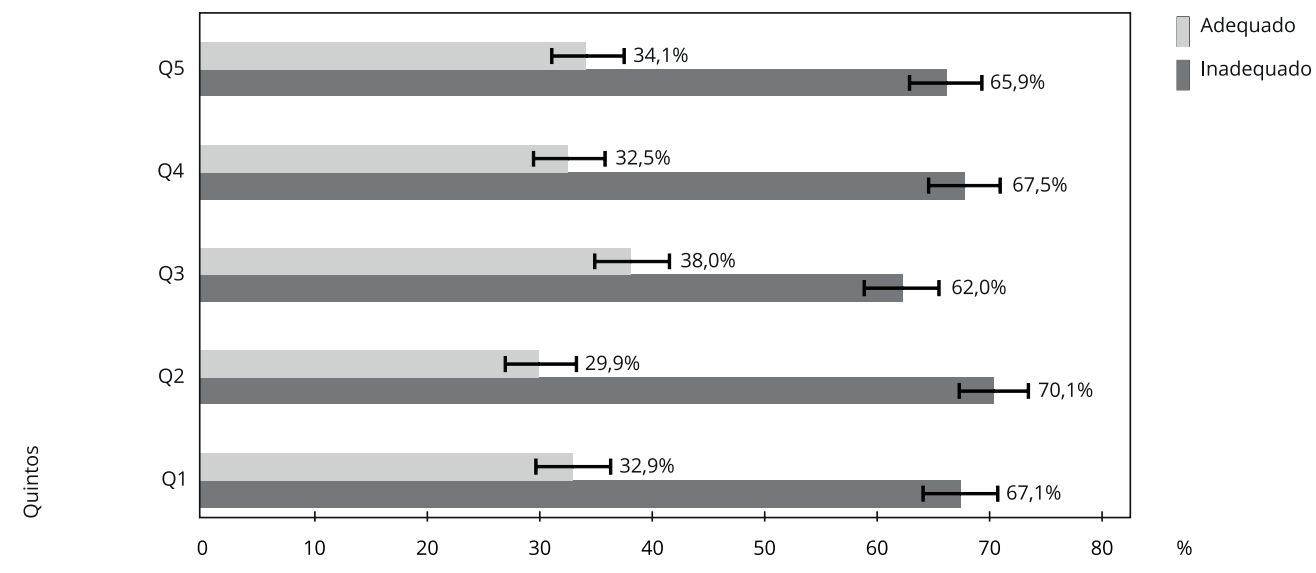

De acordo com os índices avaliados (SII: desigualdade absoluta e CIX: desigualdade relativa), foram encontradas desigualdades discretas na prevalência de ganho de peso adequado com relação à escolaridade materna e renda em quintos (Figura 1). Observou-se menor prevalência de ganho de peso adequado entre as mães com 0-4 anos de estudos e naquelas pertencentes ao quinto mais pobre (1o quinto), no entanto, as diferenças, tanto absolutas quanto relativas, identificadas para as variáveis avaliadas não foram estatisticamente significativas (Tabela 3). 
Desigualdades absolutas (Slope Index - SII) e relativas (Concentration Index - CIX) no ganho de peso gestacional adequado classificado de acordo com o Instituto de Medicina dos Estados Unidos 1 em relação à escolaridade materna e renda familiar em quintos. Coorte de nascimento 2015, Pelotas, Rio Grande do Sul, Brasil (N = 4.098).

\begin{tabular}{lllll}
\hline \multirow{2}{*}{ Variáveis } & \multicolumn{3}{c}{ Ganho de peso gestacional adequado } \\
& SII & IC95\% & CIX & IC95\% \\
\hline Escolaridade materna & 4,20 & $-0,10 ; 9,43$ & 1,66 & $-0,82 ; 4,14$ \\
Renda familiar (em quintos - 1o mais pobre e 5o mais rico) & 2,45 & $-2,06 ; 7,55$ & 1,10 & $-1,40 ; 3,58$ \\
& & & & \\
\hline
\end{tabular}

IC95\%: intervalo de 95\% de confiança.

\section{Discussão}

Neste estudo a prevalência de ganho de peso gestacional adequado foi de $30,9 \%$, sendo superior entre as com IMC pré-gestacional $<18,5 \mathrm{~kg} / \mathrm{m}^{2}$. Quando avaliado o ganho de peso gestacional considerado adequado, pode-se identificar que, mesmo que não sejam estatisticamente significativas, existem desigualdades discretas quanto à escolaridade materna e renda em quintos.

De forma geral, o ideal é que a mulher seja considerada, do ponto de vista de IMC, eutrófica no período reprodutivo e também no início da gestação e, após este período, iniciar um ganho de peso moderado e após um ano do parto retornar ao peso e IMC que tinha anteriormente à gestação 21 . Neste trabalho, os resultados foram bem consistentes mostrando que mulheres com excesso de peso pré-gestacional apresentaram maior prevalência de ganho de peso gestacional "excessivo" e as mulheres eutróficas anteriormente à gestação apresentaram prevalências de 37,9\% e 36,1\% para ganho de peso insuficiente e adequado, respectivamente. Considerando que o ganho de peso gestacional possa, em parte, ser determinado pelo estilo de vida das mulheres, um estudo de intervenção mostrou, por análise de intenção de tratar, que uma intervenção comportamental reduziu o ganho de peso gestacional "excessivo" e, ainda, aumentou os percentuais de mulheres que retornaram ao seu peso prégestacional após seis meses do parto 22.

A perda de peso após o parto é aguardada pela maioria das mulheres. No presente estudo, a média de retenção de peso foi superior após três meses do parto $(2,27 \mathrm{~kg})$ quando comparada a um ano pós-parto (1,36kg). Um estudo recente realizado com dados do National Longitudinal Survey of Youth, Estados Unidos, encontrou média de retenção de peso, de seis a 12 meses pós-parto, de 2,9kg 21, sendo superior à encontrada neste trabalho. A retenção de peso pós-parto contribui para o excesso de peso entre as mães mesmo que os motivos para esta relação ainda sejam complexos 23 . Mesmo que não exista um ponto de corte que permita avaliar quando a retenção de peso pós-parto pode ser considerada significativa, alguns estudos já têm mostrado alguma relação entre retenção de peso pós-parto e excesso de peso após um período mais longo do parto, principalmente entre mulheres com excesso de peso pré-gestacional 24,25 . No geral, a perda de peso acontece de forma mais rápida no primeiro trimestre após o parto, ficando mais lenta posteriormente 8,9, e esta relação pode ser observada no presente estudo, em que a média de retenção de peso pós-parto foi superior aos três meses quando comparada com os 12 meses após o parto.

Cabe salientar que, em termos globais, o sobrepeso e a obesidade consistem em um grave problema de saúde pública, sendo esta situação alarmante tanto em países desenvolvidos como naqueles em desenvolvimento 26. Nos últimos anos, segundo dados de uma coorte dos Estados Unidos, a obesidade pré-gestacional aumentou aproximadamente $30 \%$, sendo superior entre as mulheres de baixa renda 7 . Neste trabalho, $47 \%$ das mulheres apresentaram sobrepeso ou obesidade pré-gestacional, mostrando que metade delas poderia apresentar intercorrências durante a gestação, contribuindo também para maior indicação de parto cesariano e outras possíveis complicações tanto para a mãe quanto para a criança 27 . 
O ganho de peso gestacional adequado ainda é um desafio para muitas gestantes 27 , considerando todo o processo de mudança que ocorre neste período. As desigualdades, mensuradas pela renda e escolaridade materna, com relação ao ganho de peso considerado adequado foram discretas. No entanto, é importante destacar que o Brasil é um país marcado por importantes e persistentes desigualdades sociais em saúde 28 . Neste estudo, observou-se menor prevalência de ganho de peso adequado entre as mães menos escolarizadas ( $0-4$ anos de estudos) e entre aquelas pertencentes ao quinto mais pobre de renda (1o quinto). Um trabalho realizado nos Estados Unidos identificou que mulheres mais jovens, mais pobres, menos instruídas, ou seja, com maiores disparidades socioeconômicas, apresentaram maior probabilidade de ganho de peso excessivo durante a gestação ${ }^{29}$. Nesse mesmo sentido, um estudo recente sobre tendência e desigualdades na antropometria materna nas coortes de Pelotas (1982-2015) identificou que ao longo de 30 anos ocorreu um aumento rápido na prevalência de ganho de peso acima do recomendado entre as mulheres pertencentes ao quinto mais pobre de renda. Porém, os índices utilizados para mensurar a desigualdade existente (CIX e SII) mostram importantes declínios ao longo do tempo ${ }^{10}$. Ainda, outro estudo realizado com mais de 36 mil mulheres mostrou que as mais escolarizadas apresentaram menor risco de serem obesas ainda no início da gestação 30 .

Os achados na literatura sobre possíveis diferenças socioeconômicas com relação ao ganho de peso gestacional apresentam certa inconsistência 10,29. Uma recente revisão da literatura demonstrou que existem poucos estudos publicados avaliando a influência dos aspectos socioeconômicos no ganho de peso gestacional 31 . Nesse contexto, cabe destacar que a renda é considerada um fator importante para mensurar iniquidades socioeconômicas, uma vez que influencia em diversos aspectos de saúde, como a autonomia na escolha de uma dieta saudável durante a gestação, por exemplo 32. Ainda, o nível socioeconômico na infância pode predizer o ganho de peso gestacional e a obesidade na vida adulta 16. Mesmo que não tenham sido estatisticamente significativas, cabe destacar que as desigualdades encontradas no presente estudo salientam a importância de maior atenção para a parcela com menor renda familiar e menos escolarizada quanto à implementação de ações preventivas relacionadas ao ganho de peso, neste caso, gestacional.

Um dos aspectos positivos do estudo é pela utilização de dados de uma coorte de nascimentos com alto rigor metodológico e alta taxa de acompanhamento, além de serem realizadas análises robustas para a mensuração de desigualdades socioeconômicas. No entanto, algumas limitações devem ser mencionadas. A principal limitação deste trabalho são as perdas de informações referentes ao desfecho (ganho de peso gestacional), o que poderia modificar as prevalências encontradas, porém estimase que estas mudanças seriam muito pequenas considerando o tamanho amostral. Outra importante limitação a ser destacada é a coleta do peso pré-gestacional materno, autorreferido, podendo sofrer subestimações. No entanto, deve-se destacar que mesmo que sejam autorreferidos e possam sofrer alguns vieses, as medidas antropométricas, quando relatadas, têm alta acurácia 33, o que contribuiu para a minimização de possíveis limitações quanto ao uso em inquéritos populacionais. Ainda, cabe destacar que as mães possuíam a carteira de gestante no momento da entrevista, sendo altamente recomendado que fizessem a consulta para a confirmação dos valores referidos.

Ressalta-se que as análises de desigualdades não foram realizadas de forma convencional, considerando modelos de ajuste para as demais variáveis independentes, porém, acredita-se que para este tipo de abordagem, usada no presente trabalho, não seja necessária a estrutura analítica que deveria ser proposta caso fosse aplicado um modelo de regressão, por exemplo 20.

Este estudo encontrou desigualdades discretas quanto à renda familiar e escolaridade materna, que não se mostraram estatisticamente significativas com relação ao ganho de peso adequado. $\mathrm{O}$ presente estudo traz resultados de um país de renda média-alta, e os resultados observados por esta análise podem contribuir fortemente para a literatura que ainda não alcançou certa consistência com relação à influência socioeconômica no ganho de peso gestacional. 


\section{Colaboradores}

T. R. Flores idealizou o estudo, preparou o banco e analisou os dados, descreveu os resultados, participou da escrita do manuscrito. B. P. Nunes contribuiu para as análises dos dados e construção da discussão, revisou e aprovou a versão final do manuscrito. V. I. A. Miranda contribuiu para a escrita dos métodos, revisou criticamente o manuscrito, revisou e aprovou a versão final. M. F. Silveira e M. D. Domingues realizaram a revisão crítica do manuscrito e aprovaram a versão final. A. D. Bertoldi realizou a supervisão do trabalho, contribuiu com revisão crítica e aprovou a versão final do manuscrito.

\section{Informações adicionais}

ORCID: Thaynã Ramos Flores (0000-0003-00981681); Bruno Pereira Nunes (0000-0002-44964122); Vanessa Iribarrem Avena Miranda (00000002-9156-5036); Mariangela Freitas da Silveira (0000-0002-2861-7139); Marlos Rodrigues Domingues (0000-0002-2503-2944); Andréa Dâmaso Bertoldi (0000-0002-4680-3197).

\section{Agradecimentos}

Wellcome Trust, Conselho Nacional de Desenvolvimento Científico e Tecnológico (CNPq) e Coordenação de Aperfeiçoamento de Pessoal de Nível Superior (CAPES).

\section{Referências}

1. Institute of Medicine; National Research Council. Committee to Reexamine IOM pregnancy weight guidelines, weight gain during pregnancy: reexamining the guidelines. Washington DC: National Academy of Sciences; 2009.

2. Costa B, Paulinelli R, Barbosa M. Association between maternal and fetal weight gain: cohort study. São Paulo Med J 2012; 130:242-7.

3. Kowal C, Kuk J, Tamim H. Characteristics of weight gain in pregnancy among Canadian women. Matern Child Health J 2012; 16:66876.

4. Li C, Goran M, Kaur H, Nollen N, Ahluwalia J. Developmental trajectories of overweight during childhood: role of early life factors. Obesity 2007; 15:760-71.

5. Restall A, Taylor R, Thompson J, Flower D, Dekker G, Kenny L, et al. Risk factors for excessive gestational weight gain in a healthy, nulliparous cohort. J Obes 2014; 2014:148391.

6. Lau E, Liu J, Archer E, McDonald S, Liu J. Maternal weight gain in pregnancy and risk of obesity among offspring: a systematic review. J Obes 2014; 2014:524939.

7. Hinkle S, Sharma A, Swan D, Schieve L, Ramakrishnan U, Stein A. Excess gestational weight gain is associated with child adiposity among mothers with normal and overweight prepregnancy weight status. J Nutr 2012; 142:1851-8.

8. Olson C, Strawderman M, Hinton P, Pearson T. Gestational weight gain and postpartum behaviors associated with weight change from early pregnancy to $1 \mathrm{y}$ postpartum. Int $\mathrm{J}$ Obes 2003; 27:117-27.

9. Amorim A, Lacerda E, Kac G. Uso e interpretação dos indicadores antropométricos na avaliação do estado nutricional de gestantes. In: Kac G, Sichieri R, Gigante DP, organizadores. Epidemiologia nutricional. Rio de Janeiro: Editora Fiocruz; 2007. p. 31-47.

10. Horta B, Barros F, Lima N, Assunção M, Santos I, Domingues M, et al. Maternal anthropometry: trends and inequalities in four population-based birth cohorts in Pelotas, Brazil, 1982-2015. Int J Epidemiol 2019; 48 Suppl $1: \mathrm{i} 26-36$.

11. Nucci L, Schmidt M, Duncan B, Fuchs S, Fleck E, Britto M. Nutritional status of pregnant women: prevalence and associated pregnancy outcomes. Rev Saúde Pública 2001; 35:502-7.

12. Lipsky L, Strawderman M, Olson C. Maternal weight change between 1 and 2 years postpartum: the importance of 1 year weight retention. Obesity 2012; 20:1496-502.

13. Mannan M, Doi S, Mamun A. Association between weight gain during pregnancy and postpartum weight retention and obesity: a bias-adjusted meta-analysis. Nutr Rev 2013; 71:343-52. 
14. Chagas DC, Silva AAM, Ribeiro CCC, Batista RFL, Alves MTSSB. Efeitos do ganho de peso gestacional e do aleitamento materno na retenção de peso pós-parto em mulheres da coorte BRISA. Cad Saúde Pública 2017; 33:e00007916.

15. Huynh M, Borrell L, Chambers E. Maternal education and excessive gestational weight gain in New York City, 1999-2001: the effect of race/ethnicity and neighborhood socioeconomic status. Matern Child Health J 2013; 18:138-45.

16. Chaffee B, Abrams B, Cohen A, Rehkopf D. Socioeconomic disadvantage in childhood as a predictor of excessive gestational weight gain and obesity in midlife adulthood. Emerg Themes Epidemiol 2015; 12:4.

17. Hallal P, Bertoldi A, Domingues M, Silveira M, Demarco F, Silva I, et al. Cohort profile: the 2015 Pelotas (Brazil) Birth Cohort Study. Int J Epidemiol 2018; 47:1048-h.

18. World Health Organization. Obesity and overweight, 2016. http://www.who.int/media centre/factsheets/fs311/en/ (acessado em 25/ Jun/2016).

19. Mackenbach J, Kunst A. Measuring the magnitude of socio-economic inequalities in health: an overview of available measures illustrated with two examples from Europe. Soc Sci Med 1997; 44:757-71.

20. Barros A, Victora C. Measuring coverage in $\mathrm{MNCH}$ : determining and interpreting inequalities in coverage of maternal, newborn, and child health interventions. PLoS Med 2013; 10:e1001390.

21. Leonard S, Rasmussen K, King J, Abrams B. Trajectories of maternal weight from before pregnancy through postpartum and associations with childhood obesity. Am J Clin Nutr 2017; 106:1295-301.

22. Phelan S, Phipps M, Abrams B, Darroch F, Schaffner A, Wing R. Randomized trial of a behavioral intervention to prevent excessive gestational weight gain: the Fit for Delivery Study. Am J Clin Nutr 2011; 93:772-9.

23. Linné Y, Dye L, Barkeling B, Rössner S. Longterm weight development in women: a 15-year follow-up of the effects of pregnancy. Obes Res 2004; 12:1166-78.
24. Nehring I, Schmoll S, Beyerlein A, Hauner H, von Kries R. Gestational weight gain and longterm postpartum weight retention: a metaanalysis. Am J Clin Nutr 2011; 94:1225-31.

25. Rong K, Yu K, Han X, Szeto I, Qin X, Wang J, et al. Pre-pregnancy BMI, gestational weight gain and postpartum weight retention: a metaanalysis of observational studies. Public Health Nutr 2015; 18:2172-82.

26. Stefan K, Kantartzis K, Machann J, Schick F, Häring H. Global trends in body-mass index. Lancet 2011; 377:P1917.

27. Poston L, Caleyachetty R, Cnattingius S, Corvalán $\mathrm{C}$, Uauy $\mathrm{R}$, Herring $\mathrm{S}$, et al. Preconceptional and maternal obesity: epidemiology and health consequences. Lancet Diabetes Endocrinol 2016; 4:1025-36.

28. Paim J, Travassos C, Almeida C, Bahia L, Macinko J. Saúde no Brasil 1. O sistema de saúde brasileiro: história, avanços e desafios. Lancet 2011; 6736:11-31.

29. Endres L, Straub H, Mckinney C, Plunkett B, Minkovitz C, Schetter C, et al. Postpartum Weight Retention Risk Factors and Relationship to Obesity at One Year. Obstet Gynecol 2015; 125:144-52.

30. Heslehurst N, Ells L, Simpson H, Batterham A, Wilkinson J, Summerbell C. Trends in maternal obesity incidence rates, demographic predictors, and health inequalities in 36821 women over a 15-year period. BJOG 2007; 114:187-94.

31. Campbell E, Dworatzek P, Penava D, Vrijer B, Gilliland J, Matthews J, et al. Factors that influence excessive gestational weight gain: moving beyond assessment and counselling. J Matern Fetal Neonatal Med 2016; 29:3527-31.

32. Tarasuk V. Household food insecurity with hunger is associated with women's food intakes, health and household circumstances. J Nutr 2001; 131:2670-6.

33. Gorber S, Tremblay M, Moher D, Gorber B. A comparison of direct vs. self-report measures for assessing height, weight and body mass index: a systematic review. Obes Rev 2007; 8:307-26. 


\section{Abstract}

The study's objectives were to describe the prevalence of gestational weight gain and weight retention at 3 and 12 months postpartum and to identify possible socioeconomic inequalities in adequate gestational weight gain. A longitudinal study was performed with data from the 2015 Pelotas, Rio Grande do Sul State, Brazil, birth cohort. Gestational weight gain was classified according to the US Institute of Medicine guidelines. Weight retention at 3 and 12 months was calculated according to the mother's weight at each period, subtracting pregestational weight. To identify possible inequalities, the study used Slope Index (SII) and Concentration Index (CIX). The sample included mothers with information on the study's outcome $(n=4,102)$. Prevalence of adequate gestational weight gain was 33.5\% (95\% confidence interval - 95\%CI: 32.1; 35.0). Slight inequalities were observed in the prevalence of adequate weight gain in mothers with less schooling [CIX $=1.88$ (95\%CI: $-0.76 ; 4.52) ; S I I=4.27$ (95\%CI: -0.87 ; 9.41)] and in mothers belonging to the poorest income quintile- 1 st quintile- [CIX $=1.04$ (95\%CI: -1.60; 3.67); SII = 2.93 (95\%CI: $-2.06 ; 7.92)]$, but these differences were not statistically significant. Mean postpartum weight gain was $2.3 \mathrm{~kg}$ (standard deviation $-S D=6.4)$ and $1.4 \mathrm{~kg}(S D=8.8)$ at 3 and 12 months, respectively. One-third of the women showed adequate weight gain. The observed inequalities in prevalence of adequate gestational weight gain in women with less schooling and in the poorest income quintile were not statistically significant.

Gestational Weight Gain; Pregnancy; Body Mass Index; Socieconomic Factors

\section{Resumen}

El objetivo fue describir la prevalencia de ganancia de peso gestacional y retención de peso, tras 3 y 12 meses después del parto, así como identificar posibles desigualdades socioeconómicas en el aumento de peso gestacional adecuado. Estudio longitudinal con datos de la cohorte de nacimientos de Pelotas, Rio Grande do Sul, Brasil, de 2015. La ganancia de peso gestacional se clasificó según el Instituto de Medicina de los Estados Unidos. La retención de peso a los 3 y 12 meses se calculó considerando el peso de la madre en cada periodo sustraído del peso pregestacional. $\mathrm{Pa}$ ra identificar las posibles desigualdades se utilizó el Slope Index (SII) y el Concentration Index $(C I X)$. La muestra analítica incluyó a madres con información para el resultado en estudio ( $n$ = 4.102). La prevalencia de ganancia de peso gestacional adecuado fue de 33,5\% (intervalo de 95\% de confianza - IC95\%: 32,1; 35,0). Fueron encontradas desigualdades discretas en la prevalencia de ganancia de peso adecuado entre las menos escolarizadas [CIX = 1,88 (IC95\%: -0,76; 4,52); SII $=4,27$ (IC95\%: -0, 87; 9,41)] y, entre las madres pertenecientes al quinto más pobre-1er quinto- $[C I X=1,04$ (IC95\%: - 1,60; 3,67); SII = 2,93 (IC95\%: -2,06; 7,92)], a pesar de que esas diferencias no fueron estadísticamente significativas. La media de retención de peso pos-parto fue de $2,3 \mathrm{~kg}$ (desviación estándar $-D E=6,4$ ) y de $1,4 \mathrm{~kg}$ $(D E=8,8)$, tras 3 y 12 meses del parto, respectivamente. Un tercio de las mujeres tuvieron una ganancia de peso considerada adecuada. Las desigualdades observadas en la prevalencia de ganancia de peso gestacional adecuada, en relación con una menor escolaridad materna y el quinto más pobre de renta, no fueron estadísticamente significativas.

Ganancia de Peso Gestacional; Embarazo; Índice de Masa Corporal; Factores Socioeconómicos
Recebido em 18/Out/2019

Versão final reapresentada em 06/Abr/2020 Aprovado em 14/Abr/2020 